\title{
Cholesterol Regulates Exosome Release in Cultured Astrocytes
}

\author{
Mohammad Abdullah, Tomohisa Nakamura, Taslima Ferdous, Yuan Gao, Yuxin Chen, \\ Kun Zou and Makoto Michikawa*
}

Department of Biochemistry, Nagoya City University Graduate School of Medical Sciences, Nagoya, Japan

OPEN ACCESS

Edited by:

Rosa M. Sainz,

University of Oviedo, Spain

Reviewed by:

Heather Ferris,

University of Virginia, United States

Pedro Gonzalez-Menendez,

UMR5535 Institut de Génétique

Moléculaire de Montpellier

(IGMM), France

*Correspondence:

Makoto Michikawa

michi@med.nagoya-cu.ac.jp

Specialty section: This article was submitted to Inflammation,

a section of the journal

Frontiers in Immunology

Received: 11 June 2021 Accepted: 23 September 2021

Published: 14 October 2021

Citation:

Abdullah M, Nakamura T, Ferdous T,

Gao Y, Chen Y, Zou K and

Michikawa M (2021) Cholesterol

Regulates Exosome Release in

Cultured Astrocytes.

Front. Immunol. 12:722581.

doi: 10.3389/fimmu.2021.722581
Exosomes are vesicles secreted by various kinds of cells, and they are rich in cholesterol, sphingomyelin (SM), phosphatidylcholine, and phosphatidylserine. Although cellular sphingolipid-mediated exosome release has been reported, the involvement of other lipid components of cell membranes in the regulation of exosome release is poorly understood. Here, we show that the level of exosome release into conditioned media is significantly reduced in cultured astrocytes prepared from apolipoprotein $E$ (ApoE) knockout mice when compared to those prepared from wild-type (WT) mice. The reduced level of exosome release was accompanied by elevated levels of cellular cholesterol. The addition of cholesterol to WT astrocytes significantly increased the cellular cholesterol levels and reduced exosome release. PI3K/Akt phosphorylation was enhanced in ApoEdeficient and cholesterol-treated WT astrocytes. In contrast, the depletion of cholesterol in ApoE-deficient astrocytes due to treatment with $\beta$-cyclodextrin recovered the exosome release level to a level similar to that in WT astrocytes. In addition, the reduced levels of exosome release due to the addition of cholesterol recovered to the control levels after treatment with a PI3K inhibitor (LY294002). The cholesterol-dependent regulation of exosome release was also confirmed by in vivo experiments; that is, exosome levels were significantly reduced in the CSF and blood serum of WT mice that were fed a high-fat diet and had increased cholesterol levels when compared to those in WT mice that were fed a normal diet. These results suggest that exosome release is regulated by cellular cholesterol via stimulation of the PI3K/Akt signal pathway.

Keywords: apolipoprotein E, astrocytes, cholesterol, exosome, PI3K, Akt

\section{INTRODUCTION}

The fusion of multivesicular bodies with the plasma membrane causes the release of intraluminal vesicles within the multivesicular bodies into the extracellular milieu as exosomes $(1,2)$. These membrane-bound macrovesicles measure 30 to $100 \mathrm{~nm}$ in diameter (3), and they contain proteins (4), lipids (5-7), mRNAs, and microRNAs (8-10). Exosomes have been isolated and purified from biological fluids, such as urine (4), blood $(11,12)$, and breast milk (13), and from in vitro cultures of numerous cell types $(5,6,14)$. Their presence and stability in biological fluids, as well as their particular composition, make exosomes a potential source of biomarkers for several diseases $(12,15)$. 
Recent studies have shown the involvement of several proteins and lipids in the biogenesis and release of exosomes. For example, it has been shown that endosomal sorting complexes required for transport proteins $(16,17)$ and lipidrelated proteins, such as phospholipid- and phosphoinositidespecific phospholipase C (18), play a role in exosome secretion. It is reasonable to assume that lipids in the cell membrane may affect exosome release since exosomes are released from cells after the fusion of multivesicular bodies with the plasma membrane, and they are rich in cholesterol, sphingomyelin (SM), phosphatidylcholine, and phosphatidylserine when compared to the donor cells (7). From this point of view, studies have demonstrated that exosome release is mediated by cellular lipids, such as SM and ceramide $(19,20)$. However, the function of other lipids, such as cholesterol, one of the major lipid components of the cellular membrane, in the regulation of exosome release remains unknown.

Regarding lipid transport in the brain, apolipoprotein $\mathrm{E}$ (ApoE) has been shown to be involved in cholesterol transport $(21,22)$. In the central nervous system, ApoE is one of the major lipid acceptors (23), and it interacts with ATP-binding cassette A1 (24) to remove cholesterol from cells to generate high-density lipoprotein particles (25) in an ApoE-isoform-specific manner $(22,26,27)$. Altered ApoE isoform-specific high-density lipoprotein formation and its supply to neural cells via ApoE receptors may be causes of the altered cholesterol metabolism in the Alzheimer's disease (AD) brain. In line with this notion, it is known that the ApoE-knock-out (KO) mouse is a model for atherosclerosis. These lines of evidence led us to perform a study to determine the effect of cholesterol and its transporter, ApoE, on exosome release in cultured astrocytes and in vivo.

\section{MATERIALS AND METHODS}

\section{Animals}

WT C57BL/6J mice were obtained from SLC, Inc. (Shizuoka, Japan). ApoE3-KO C57BL/6J mice were purchased from Charles River Laboratories Japan, Inc. (Yokohama, Japan). All mice were bred on a 12-h light-dark schedule with ad libitum access to standard chow (CE-2, CLEA, Shizuoka, Japan) and tap water.

\section{Cell Culture}

Primary cultures of mixed glial cells were prepared from the brain of C57BL/6 mouse pups on postnatal day 1 as described previously $(14,22,26)$. Briefly, isolated cortices of the brain were minced, and the cortical fragments were incubated in $0.25 \%$ trypsin and $20 \mu \mathrm{g} / \mathrm{ml}$ DNase I in phosphate-buffered saline (8.1 mM Na2 $\mathrm{HPO}_{4}, 1.5 \mathrm{mM} \mathrm{KH} 2 \mathrm{PO}_{4}, 137 \mathrm{mM} \mathrm{NaCl}$, and 2.7 $\mathrm{mM} \mathrm{KCl}, \mathrm{pH} 7.4$ ) at $37^{\circ} \mathrm{C}$ for $15 \mathrm{~min}$. The fragments were then dissociated by pipetting to produce single-cell suspensions. The dissociated cells were seeded into a $75-\mathrm{cm}^{2}$ flask at a cell density of $1 \times 10^{7}$ in Dulbecco's Modified Eagle Medium (DMEM) containing $10 \%$ fetal bovine serum, at which point microglia were removed by shaking. After 10 days of incubation in vitro, astrocytes in the monolayer were trypsinized $(0.1 \%)$ and reseeded onto $6-\mathrm{cm}^{2}$ dishes. The astrocyte-rich cultures were maintained in DMEM containing 10\% fetal bovine serum until use.

\section{Cholesterol Depletion and Drug Treatments}

A stock solution of methyl- $\beta$-CD (Sigma Aldrich, St. Louis, MO, USA) was prepared by dissolving $\beta-C D$ in MilliQ water to a concentration of $16.5 \mathrm{mM}$. For cholesterol depletion, astrocytes were treated with $\beta-\mathrm{CD}$ at a final concentration of $0.1,1$, or $2 \mathrm{mM}$ for $24 \mathrm{~h}$ at $37^{\circ} \mathrm{C}$. Cholesterol (Wako, Osaka, Japan) was dissolved in $100 \%$ ethanol to prepare stock solutions with a concentration of $10 \mathrm{mg} / \mathrm{ml}$; these solutions were subsequently diluted and used at a final concentration of 5 or $10 \mu \mathrm{M}$. LY294002 (Calbiochem, Tokyo, Japan), a PI3K inhibitor, was dissolved in dimethyl sulfoxide to prepare stock solutions with a concentration of $10 \mathrm{mM}$; these solutions were subsequently diluted and used at a final concentration of 10 or $20 \mu \mathrm{M}$. For each experiment, the culture medium was replaced with DMEM containing the indicated amount of drug without serum, and incubated for the indicated time.

\section{Western Blot Analysis}

The CM were collected, and cells were washed in cold phosphatebuffered saline two times, then collected with a scraper in $100 \mu \mathrm{l}$ of radioimmunoprecipitation assay buffer containing a cocktail of protease inhibitors purchased from Roche Diagnostics (Indianapolis, IN, USA) and phosphatase inhibitors (Wako, Tokyo, Japan). The cell lysates were transferred to a $1.5-\mathrm{ml}$ microtube and homogenized on ice with a glass homogenizer. The resultant homogenate was centrifuged at $12,000 \times \mathrm{rpm}$ for $5 \mathrm{~min}$ at $4^{\circ} \mathrm{C}$ to separate the solution from the pellet fraction. The protein concentrations in the supernatants were determined by a BCA protein assay kit (Pierce, Rockford, IL, USA). An equal volume of $\mathrm{CM}$ and equal amount of homogenate protein were mixed with the sampling buffer (100 mM Tris- $\mathrm{HCl}$ ( $\mathrm{pH} 7.4), 10 \%$ glycerol, $4 \%$ sodium dodecyl sulfate, $10 \%$ mercaptoethanol, and $0.01 \%$ bromophenol blue), and analyzed by $12.5 \%$ Tris/glycine sodium dodecyl sulfate-polyacrylamide gel electrophoresis. The separated proteins were electrophoretically transferred onto polyvinylidene difluoride membranes, Immobilon-P (MerckMillipore, Carrigtwohill, IRL), using a transfer buffer $(0.1 \mathrm{M}$ Tris, $0.192 \mathrm{M}$ glycine, and 10\% methanol). Membranes were then incubated in a blocking solution consisting of $5 \%$ powdered milk in Tris-buffered saline with Tween $20(10 \mathrm{mmol} /$ L Tris- $\mathrm{HCl}$ (pH 8.0), $150 \mathrm{mmol} / \mathrm{L} \mathrm{NaCl}$, and $0.1 \%$ Tween 20) for $1 \mathrm{~h}$ at room temperature. These membranes were then incubated with the respective antibodies at $4^{\circ} \mathrm{C}$ overnight. The primary antibodies used were rabbit anti-flotillin 1 polyclonal antibody (Sigma Aldrich), mouse anti-HSP90 monoclonal antibody (BD Bioscience, Franklin Lakes, NJ, USA), rabbit anti-ApoE polyclonal antibody (EMD Millipore, Billerica, MA, USA), rabbit anti-Akt polyclonal antibody (Cell Signaling, Beverly, MA, USA), rabbit anti-phospho-Akt polyclonal antibody (Cell Signaling), rabbit anti-PI3K monoclonal antibody (Cell Signaling), rabbit 
anti-phospho-PI3K polyclonal antibody (Cell Signaling), and mouse anti- $\alpha$-tubulin monoclonal antibody (Sigma Aldrich). The membranes were washed, then incubated with the appropriate secondary antibody conjugated to horseradish peroxidase. Immunoreactive bands were visualized with ImmunoStar Zeta or ImmunoStar LD (Wako, Osaka, Japan), and analyzed with the Amersham Imager 680 (GE Healthcare Life Science, Marlborough, MA, USA). Signal intensities were quantified by ImageJ (1.46r; Java 1.6.0-20 [64 bit] (NIH, Bethesda, MD, USA).

\section{ELISA for the Determination of the Exosome Levels}

Serum exosome levels were determined by a CD9/CD63 exosome ELISA kit (Cosmo Bio., Tokyo, Japan), which is a sandwich ELISA kit that uses anti-CD63 antibody as a capture antibody and anti-CD9 antibody as a detection antibody, according to the manufacturer's instructions.

\section{Determination of the Cholesterol Levels}

For the analysis of the cellular cholesterol levels, $1 \mathrm{ml}$ of each lysate solution was transferred into separate clean glass tubes containing $4.0 \mathrm{ml}$ of chloroform/methanol $(2: 1 \mathrm{v} / \mathrm{v})$. The organic phase was separated from the aqueous phase by centrifugation at 3,000 rpm and $4^{\circ} \mathrm{C}$ for $15 \mathrm{~min}$, then the cholesterol-containing phase was removed from the bottom to another tube and dried under $\mathrm{N}_{2}$ gas. The dried cholesterol was then dissolved in $300 \mu \mathrm{l}$ of isopropyl alcohol, and each sample was transferred onto 96well polypropylene plates (Corning Gorilla Glass, Corning, NY, USA) and dried under air flow. The dried content of cholesterol was determined by the Amplex ${ }^{\circledR}$ Red Cholesterol Assay Kit (Life Technologies, Eugene, OR, USA).

\section{Collection of CSF, Blood Serum, and Brain Specimens From Mice}

Three-month-old WT mice were fed a high-fat diet or a control diet for four months. All mice were housed under a 12-h dark/ light cycle, and had access to the high-fat diet or control diet and water ad libitum. At the age of 7 months, the mice were sacrificed by anesthesia. Three mixtures of combination anesthetics were prepared with $0.3 \mathrm{mg} / \mathrm{kg}$ of medetomidine (Medetomin, Meiji Seika Pharma Co., Ltd., Tokyo, Japan), 4.0 mg/kg of midazolam (Dormicum, Maruishi Pharmaceutical Co., Ltd., Osaka, Japan), and $5.0 \mathrm{mg} / \mathrm{kg}$ of butorphanol (Vetorphale, Meiji Seika Pharma Co., Ltd.), and the volume was adjusted with sterilized saline. The anesthetics were administered to mice by intraperitoneal injection at a volume of $0.01 \mathrm{ml} / \mathrm{g}$ of body weight. For the investigations of the extracellular exosome and cholesterol levels, CSF from the cisterna magna, blood serum, and brain were collected from the mice.

\section{Statistical Analysis}

Immunopositive bands that were visualized by western blot analysis were quantified by image analysis software (ImageJ 1.46r; Java 1.6.0-20 [64 bit]). Results are expressed as the mean \pm standard error (SE) of three independent experiments.
Statistical analysis was performed using one-way analysis of variance (ANOVA) followed by the Bonferroni-Dunn test and Student's $t$-test.

\section{RESULTS}

Firstly, to determine the effect of ApoE deficiency on exosome release from cultured astrocytes into serum-free media, we prepared astrocyte cultures from wild-type (WT) and ApoE-KO mice. Western blot analysis was performed using conditioned media (CM) collected from astrocytes that were cultured for $48 \mathrm{~h}$ in serum-free media. When flotillin and heat shock protein 90 (HSP90) were used as exosome markers, we found that exosome release in the $\mathrm{CM}$ was significantly reduced in ApoE-deficient astrocytes when compared to WT astrocytes (Figures 1A, B). However, the cellular levels of these proteins remained unchanged (Figures 1C, D). The cellular cholesterol levels in ApoE-deficient astrocytes were significantly increased when compared to those in WT astrocytes (Figure 1E). Since it has been shown that cellular cholesterol regulates exosome release by modulating PI3K and Akt activity $(28,29)$, we examined the phosphorylation levels of PI3K and Akt. The results showed that the phosphorylation levels of PI3K and Akt were significantly increased in ApoE-deficient astrocytes when compared to WT astrocytes, whereas the total levels of these proteins remained unchanged between ApoEdeficient and WT astrocytes (Figures 1F, G). We examined this is also the case for in vivo. The serum collected from WT and ApoE-KO mice were subjected to western blot analysis using antiflotillin and HSP90 antibodies. The levels of flotillin in the serum from ApoE-KO mice was significantly lower than those in the serum from WT mice. The levels of HSP90 shows lower tendency in the serum from ApoE-KO mice compared with those from WT mice (Figures 1H, I).

To determine whether these changes were caused by cholesterol, we further analyzed the cholesterol-dependent regulation of exosome secretion from cultured astrocytes. Astrocyte cultures prepared from ApoE-KO and WT mice were treated with cholesterol at a concentration of 0,5 , or 10 $\mu \mathrm{M}$. In WT astrocytes, treatment with cholesterol at the concentrations of 5 and $10 \mu \mathrm{M}$ significantly reduced the secreted levels of flotillin and HSP90 to levels similar to those of ApoE-deficient astrocyte without cholesterol treatment (Figures 2A-C), and there were no changes in the cellular levels of flotillin and HSP90 (Figures 2A, D, E). When the WT astrocytes were treated with 5 or $10 \mu \mathrm{M}$ cholesterol, the cellular cholesterol levels increased to levels similar to those of ApoEdeficient astrocytes without cholesterol treatment (Figure 2F). In WT astrocytes, along with the increase in cellular cholesterol levels, the levels of exosome release, as determined by the flotillin and HSP90 levels, were reduced to levels similar to those of ApoE-deficient astrocytes without cholesterol treatment (Figures 2B, C, F). Interestingly, levels of ApoE released from WT astrocytes remained unchanged when the cultures were tread with cholesterol, suggesting that cellular cholesterol has no effect on generating ApoE-containing particles (Figure 2A). 


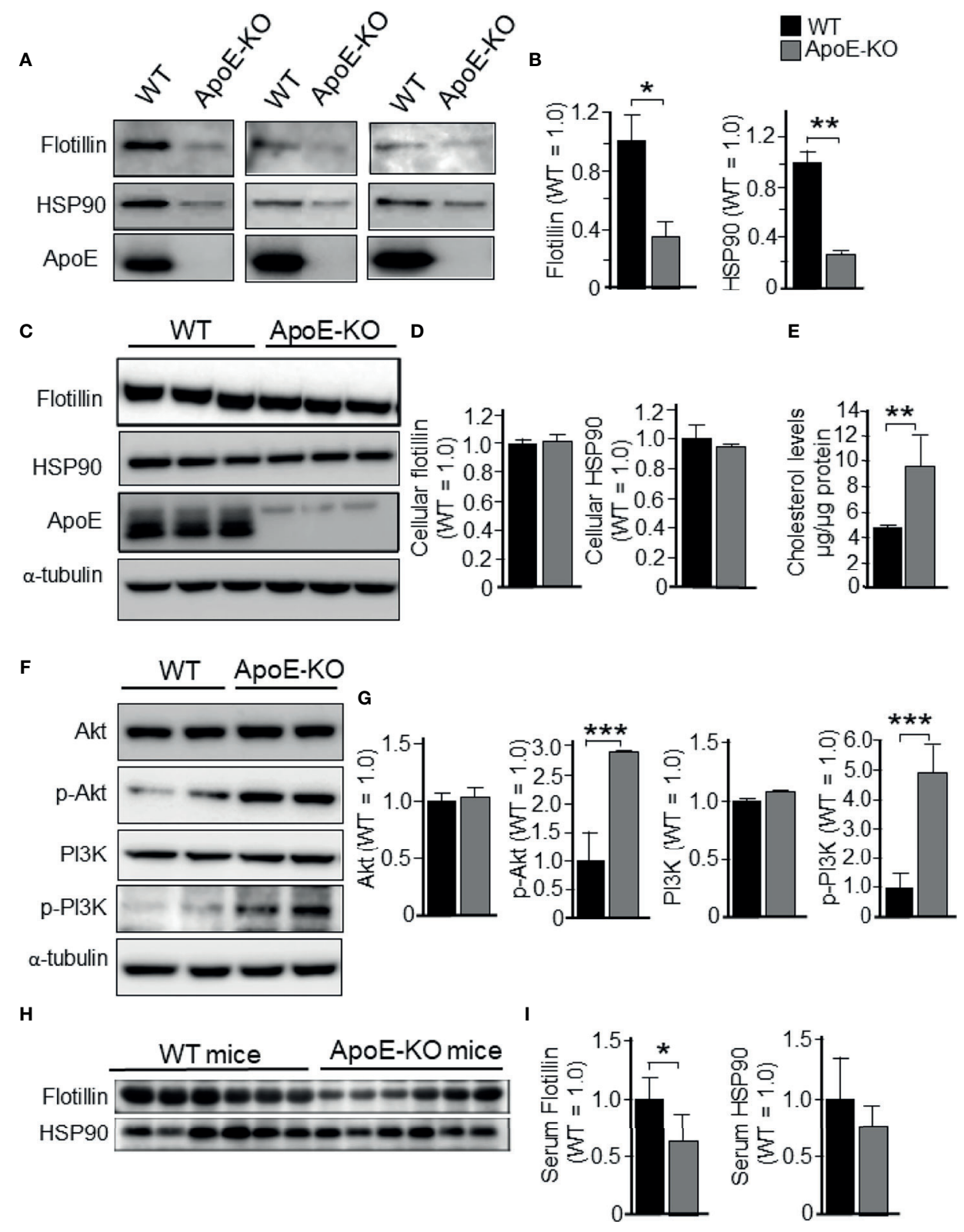

FIGURE 1 | Levels of exosome release in cultured astrocytes prepared from WT and ApoE-KO mouse brain. Astrocyte-rich cultures were prepared from mouse brains as described in the Experimental Procedures section. CM and cell lysates were harvested and subjected to western blot analysis using antibodies against flotillin, HSP90, p-PI3K, pan-PI3K, p-Akt, pan-Akt, and ApoE. WT and ApoE-deficient astrocytes were cultured for $48 \mathrm{~h}$, then the CM (A, B) and cell lysates (C, D) were obtained and analyzed by western blot analysis. (E) The cellular cholesterol levels were determined by a cholesterol determination kit. (F, G) Cellular levels of p-PI3K, pan-PI3K, p-Akt, and pan-Akt were determined by western blot analysis using specific antibodies against these molecules. (H, I) Flotillin and HSP90 levels in the serum collected from WT and ApoE-KO mice were subjected to western blot analysis using anti-flotillin and HSP90 antibodies. The intensity of each band was quantified by densitometry. Data are expressed as the mean \pm SE. $\mathrm{n}=3$ each. ${ }^{*} p<0.05,{ }^{\star *} p<0.01,{ }^{\star \star *} p<0.001$ by Student's $t$-test.

We next determined whether a higher cholesterol level in cells is responsible for the activation of PI3K/Akt. We found that the addition of cholesterol at concentrations of 5 and $10 \mu \mathrm{M}$ to WT astrocyte cultures significantly increased the levels of phosphorylated PI3K/Akt when compared to WT astrocyte cultures without the addition of cholesterol (Figures 3A, C, E). However, cholesterol treatment had no effect on the levels of phosphorylated PI3K/Akt in ApoE-deficient astrocytes (Figures 3A, C, E). With respect to the total PI3K/Akt (pan$\mathrm{PI} 3 \mathrm{~K} / \mathrm{Akt}$ ), treatment with cholesterol had no effect in ApoEdeficient astrocytes or WT astrocytes (Figures 3B, D).

To determine whether higher cholesterol levels lead to reduced exosome release, we carried out experiments to determine the effect of cholesterol depletion by treatment with 

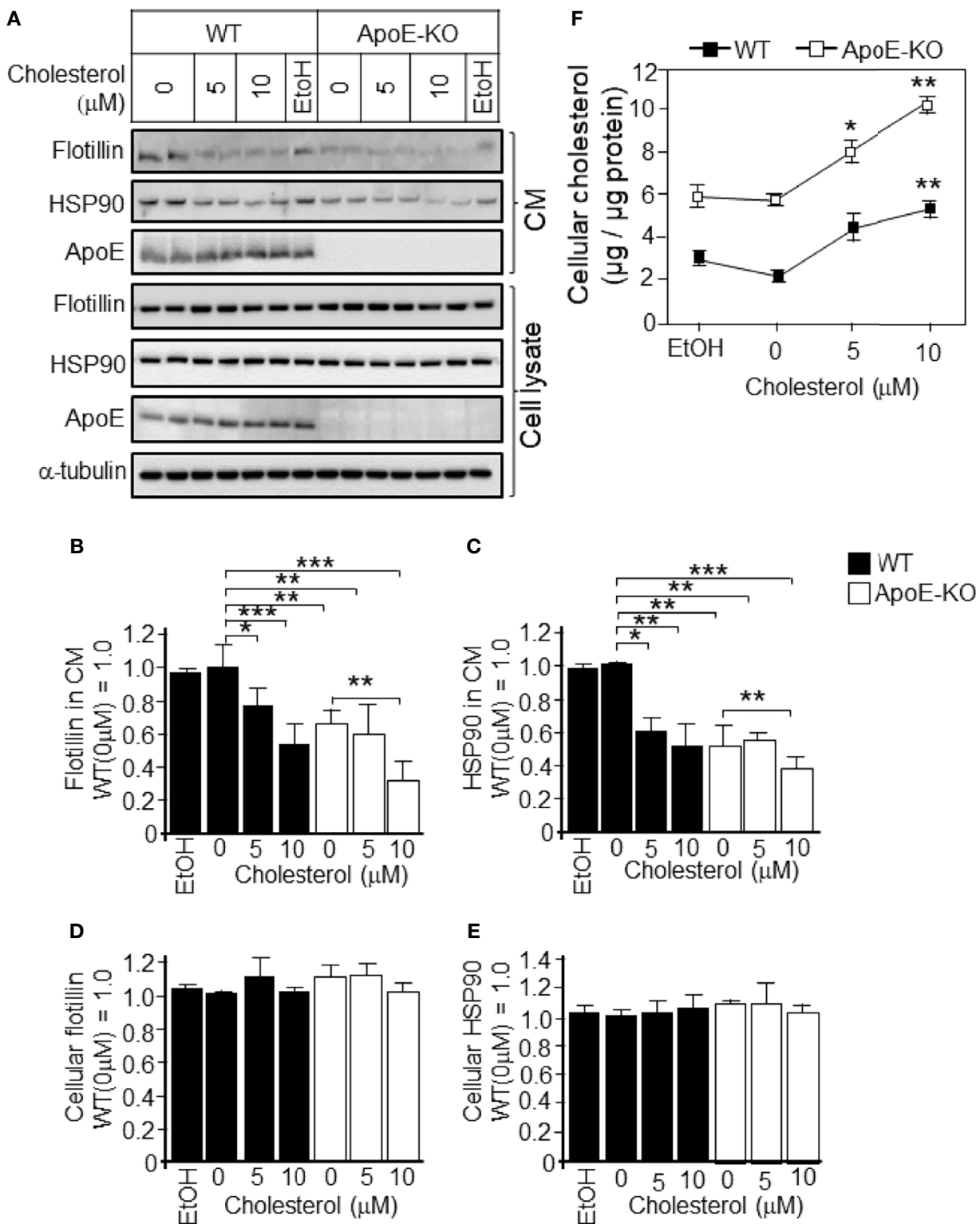

FIGURE 2 | Levels of exosome release in cultured WT and ApoE-deficient astrocytes treated with varying concentrations of cholesterol. Primary astrocyte cultures prepared from WT and ApoE-KO mouse brain were treated with cholesterol at the concentration of 0,5 , or $10 \mu \mathrm{M}$. The cultures were then incubated for $48 \mathrm{~h}$, and the CM and cell lysates were harvested. The samples were subjected to western blot analysis using antibodies against flotillin, HSP90, ApoE, and $\alpha$-tubulin. (A) Western blot analysis of the CM and cell lysates. (B-E) The signal intensities of the western blots were quantified. (F) The cellular cholesterol levels were determined by a cholesterol determination kit. Data are expressed as the mean $\pm \mathrm{SE}$. $\mathrm{n}=3 .{ }^{*} p<0.05,{ }^{* *} p<0.01,{ }^{\star \star *} p<0.001$ by one-way ANOVA followed by the Bonferroni-Dunn test versus EtOH.

$\beta$-cyclodextrin ( $\beta$-CD) on exosome release, as demonstrated by flotillin/HSP90 release. Astrocytes were treated with $\beta-\mathrm{CD}$ at a final concentration of $0.1,1$, or $2 \mathrm{mM}$ for $24 \mathrm{~h}$ at $37^{\circ} \mathrm{C}$. The cellular cholesterol levels of astrocytes isolated from ApoE-KO and WT mice were significantly reduced in a $\beta$-CD-dosedependent manner (Figure 4F). In both types of cultured astrocytes, exosome release increased when the cellular cholesterol level decreased, and the difference in exosome release between ApoE-deficient astrocytes and WT astrocytes reached similar levels with the treatment of $\beta-\mathrm{CD}$ at the concentrations of $0.1,1$, and $2 \mathrm{mM}$ (Figures $4 \mathrm{~A}-\mathrm{C}$ ). In contrast, the cellular levels of flotillin and HSP90 remained unchanged (Figures 4A, D, E). These results indicated that reduced cholesterol levels induce exosome release.

We further analyzed the effect of cholesterol depletion on cell signaling molecules. We observed that the astrocytes with lower levels of cholesterol had significantly reduced levels of the phosphorylated form of PI3K/Akt when compared to the 

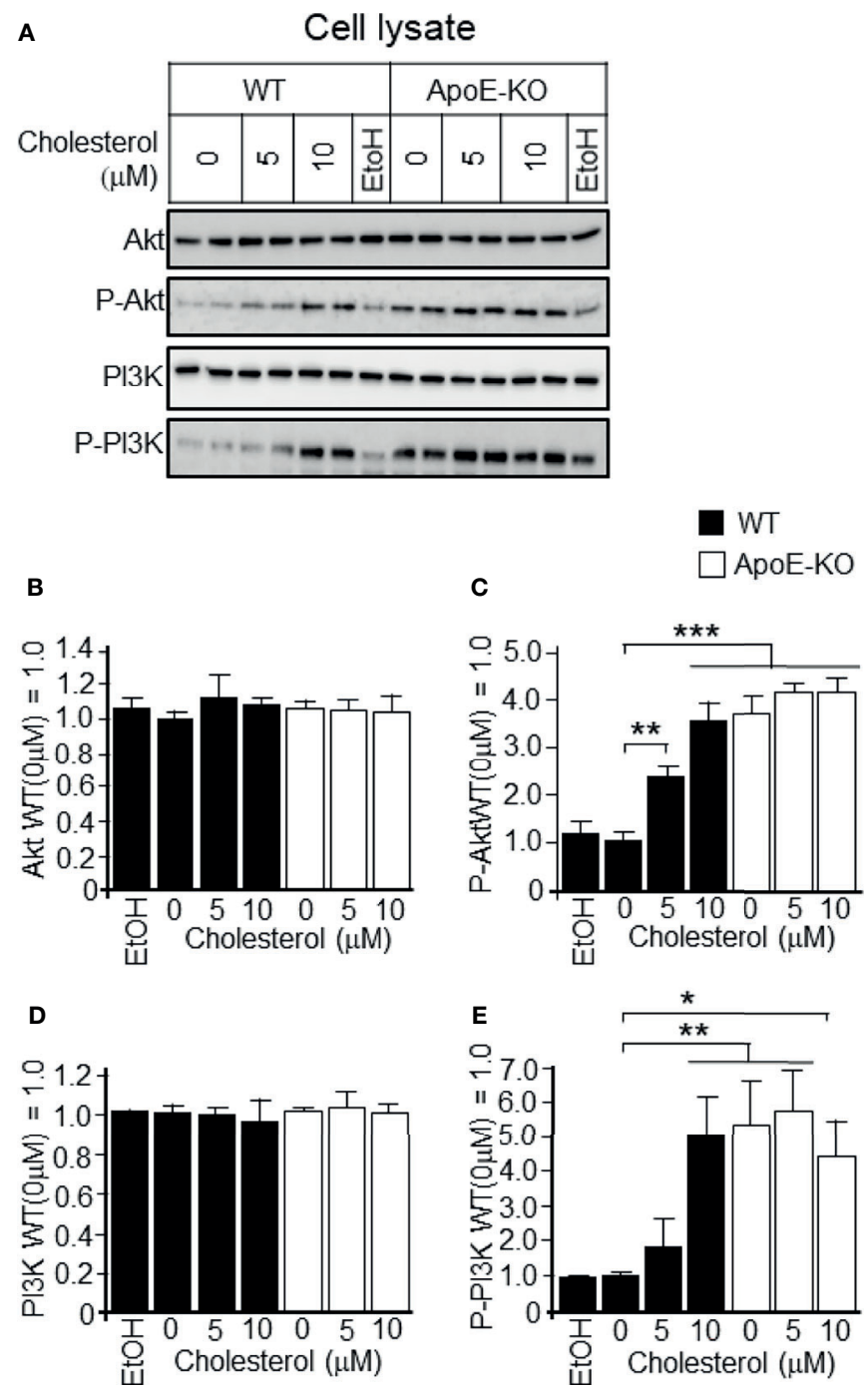

FIGURE 3 | Levels of p-Akt, pan-AKT, p-PI3K, and pan-PI3K in WT and ApoE-deficient astrocytes treated with varying concentrations of cholesterol. Primary astrocyte cultures prepared from WT and ApoE-KO mouse brain were treated with cholesterol at the concentration of 0 , 5 , or $10 \mu \mathrm{M}$. The cultures were then incubated for $48 \mathrm{~h}$, and the cell lysates were harvested. (A) The cell lysates were subjected to western blot analysis using antibodies against Akt, p-Akt, PI3K, and p-PI3K. (B-E) The intensity of each band was quantified by image analysis software (ImageJ 1.46r; Java 1.6.0-20 [64 bit]). Data are expressed as the mean \pm SE. $\mathrm{n}=3 .{ }^{*} p<0.05,{ }^{* *} p<0.01,{ }^{* * *} p<0.001$ by one-way ANOVA followed by the Bonferroni-Dunn test.

control astrocytes without $\beta$-CD treatment (Figures 5A, C, E). However, there were no significant changes in the levels of total PI3K/Akt (pan-PI3K/Akt; Figures 5B, D).

To determine whether the increased levels of $\mathrm{p}-\mathrm{PI} 3 \mathrm{~K} / \mathrm{p}$-Akt that were induced by higher levels of cellular cholesterol were responsible for the reduced exosome release, we treated astrocytes with PI3K-specific inhibitor LY294002 at the concentration of 10 or $20 \mu \mathrm{M}$. We confirmed that LY294002 treatment significantly reduced the p-PI3K and p-Akt levels in ApoE-deficient and WT astrocytes (Figures 6A, C, E); however, it did not have any effect on the total PI3K and Akt levels in either type of astrocytes (Figures 6B, D). We also examined whether the PI3K/Akt pathway is responsible for the regulation of exosome release. In the presence of a PI3K inhibitor,
LY294002, the decreased levels of exosome release, as demonstrated by the flotillin and HSP90 release, in ApoEdeficient astrocytes, which have higher levels of cellular cholesterol, recovered without any changes in the cellular flotillin and HSP90 levels (Figures 7A-F). These results suggested that exosome release is regulated by cellular cholesterol via the PI3K/Akt signaling pathway.

To investigate whether the cholesterol-dependent exosome release that was seen in cultures is also seen in vivo, 3-month-old WT mice were fed a high-fat diet or a control diet for 4 months. The basic formulation of a high-fat diet and a control diet are shown in Tables 1. At 7 months of age, the mice were sacrificed, and CSF from the cisterna magna, blood serum, and brain were collected. The samples were subjected to western blot analysis. 
A

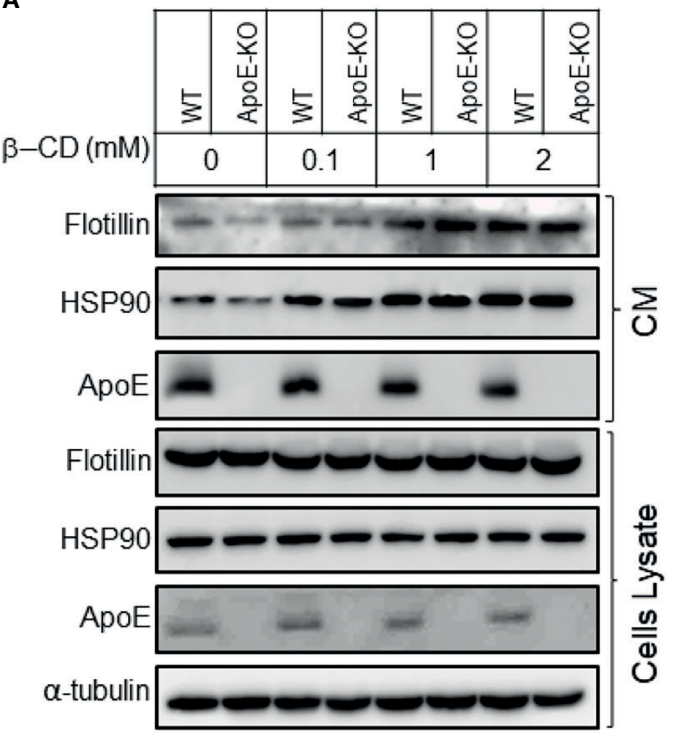

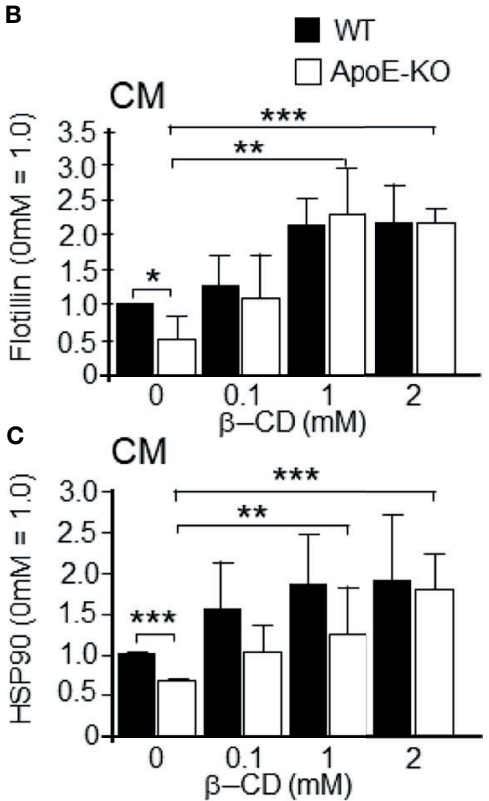

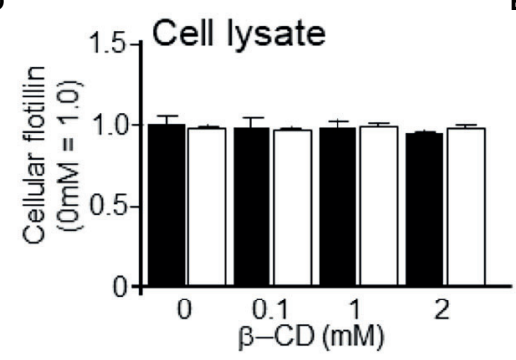

E
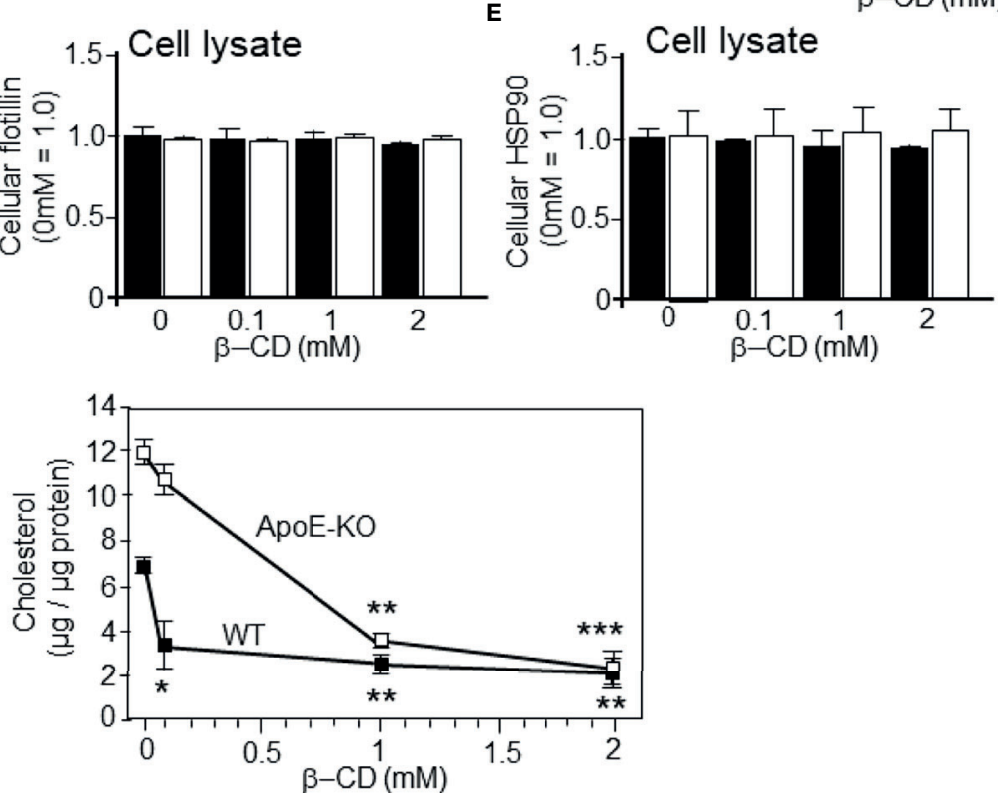

FIGURE 4 | Effect of cholesterol depletion due to $\beta$-CD on exosome release. Primary astrocyte cultures prepared from WT and ApoE-KO mouse brain were treated with $\beta-C D$ at the concentration of $0.1,1$, or $2 \mathrm{mM}$, and incubated for $24 \mathrm{~h}$. Then, the $\mathrm{CM}$ and cell lysates were harvested and subjected to western blot analysis to determine the flotillin, HSP90, and ApoE levels in the CM (A-C) and cell lysates (A, D, E). (B-E) The intensity of each band was quantified by image analysis software (ImageJ 1.46r; Java 1.6.0-20 [64 bit]]). Data are expressed as the mean \pm SE. $n=3$ each. ${ }^{*} p<0.05,{ }^{* *} p<0.01,{ }^{* *} p<0.001$ by one-way ANOVA followed by the Bonferroni-Dunn test. (F) The cellular cholesterol levels were determined by a cholesterol determination kit. Data are expressed as the mean \pm SE. $n=3$ each. ${ }^{*} p<0.05,{ }^{* *} p<0.01,{ }^{* \star *} p<0.001$ by one-way ANOVA followed by the Bonferroni-Dunn test versus no $\beta$-CD treatment.

We found that the levels of exosomes, as demonstrated by the flotillin and HSP90 levels, in CSF were significantly lower in the mice that were fed the high-fat diet than in those that were fed the control diet (Figures 8A-C). The cholesterol levels in brain were higher in the mice that were fed the high-fat diet than in those that were fed the control diet (Figure 8D). We also found that the serum levels of exosomes, as demonstrated by the flotillin and HSP90 levels, were significantly lower in the mice that were fed the high-fat diet than in those that were fed the control diet (Figures 8E-G). The levels of serum cholesterol were significantly higher in the mice that were fed the high-fat diet than in those that were fed the control diet (Figure 8H). We also analyzed the serum exosome levels using a CD9/CD63 exosome enzyme-linked immunosorbent assay (ELISA) kit. In accord with the results of the western blot analysis, the ELISA revealed that the serum exosome levels were significantly lower in the mice 


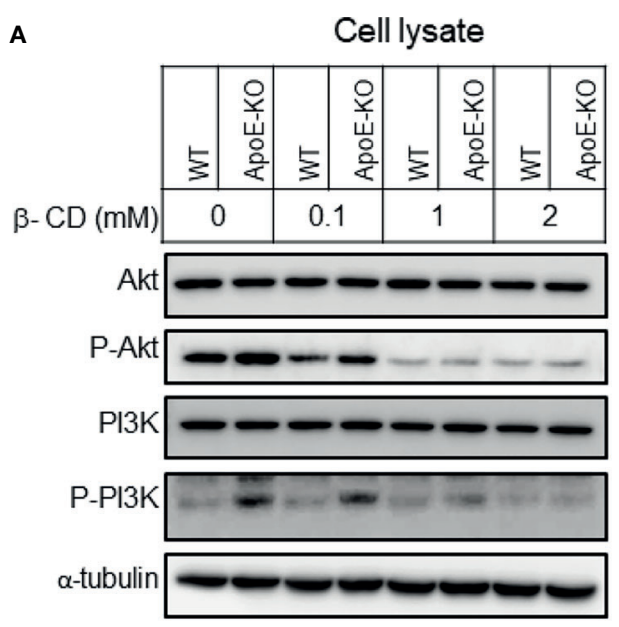

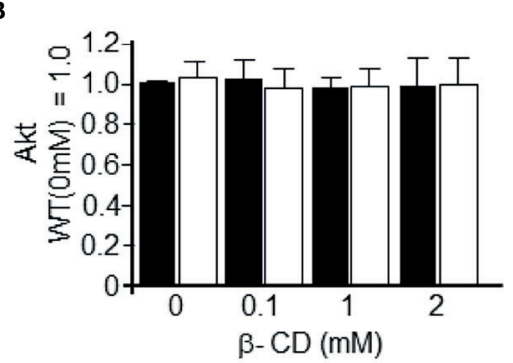

D

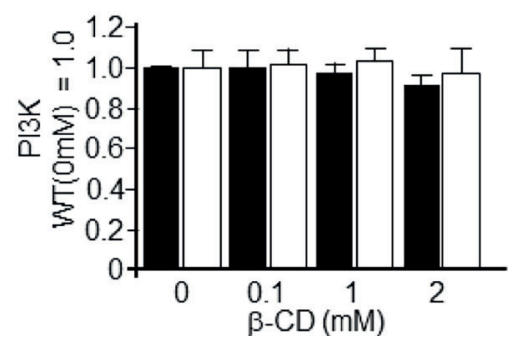

WT

ApoE-KO

C

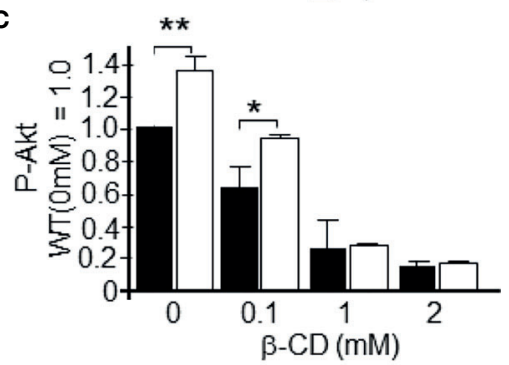

E

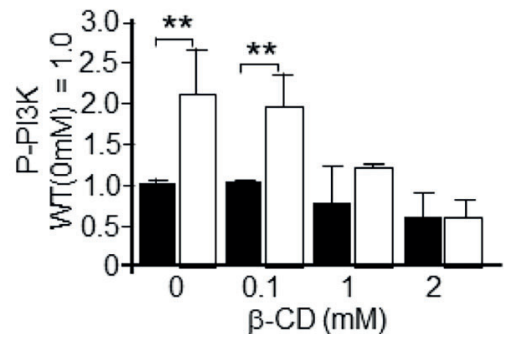

FIGURE 5 | Effect of cholesterol depletion due to $\beta-C D$ on phospho-PI3K and phospho-Akt. Astrocyte cultures prepared from WT and ApoE-KO mouse brain were treated with $\beta-C D$ at the concentration of $0.1,1$, or $2 \mathrm{mM}$, and incubated for $24 \mathrm{~h}$. Then, the $\mathrm{CM}$ and cell lysates were harvested and subjected to western blot analysis. (A) Western blot analysis was performed using antibodies against Akt, p-Akt, PI3K, p-PI3K, and $\alpha$-tubulin as an internal control. (B-E) The intensity of each band was quantified by image analysis software (ImageJ 1.46r; Java 1.6.0-20 [64 bit]). Data are expressed as the mean \pm SE. $n=3$ each. ${ }^{\star} p<0.05,{ }^{* *} p<0.01$ by one-way ANOVA followed by the Bonferroni-Dunn test.

that were fed the high-fat diet than in those that were fed the control diet (Figure 8I). ApoE levels in the CSF collected from mice fed with control diet and high-fat diet were determined by western blot analysis using anti-ApoE antibody. The ApoE levels in the CSF of these mice show similar levels (Figures 8J, K).

\section{DISCUSSION}

In this study, we found that exosome release into the CM was significantly reduced in cultured astrocytes prepared from ApoEKO mice when compared to those from WT mice. We also found that the decreased levels of exosome release in ApoE-deficient astrocytes were accompanied by increased levels of cellular cholesterol as well as phosphorylated PI3K and Akt. When the cellular cholesterol levels increased due to the treatment with cholesterol in astrocytes prepared from WT mice, the levels of exosome release decreased, while the levels of phosphorylated PI3K and Akt increased. The decreased levels of exosomes and the increased levels of cholesterol recovered to the control levels in ApoE-deficient astrocytes as a result of cholesterol depletion due to $\beta$-CD. Similarly, the decreased levels of exosomes and the increased levels of cholesterol recovered to the control levels in ApoE-deficient astrocytes as a result of treatment with the PI3K inhibitor, LY-294002. Moreover, we found that exosome release 


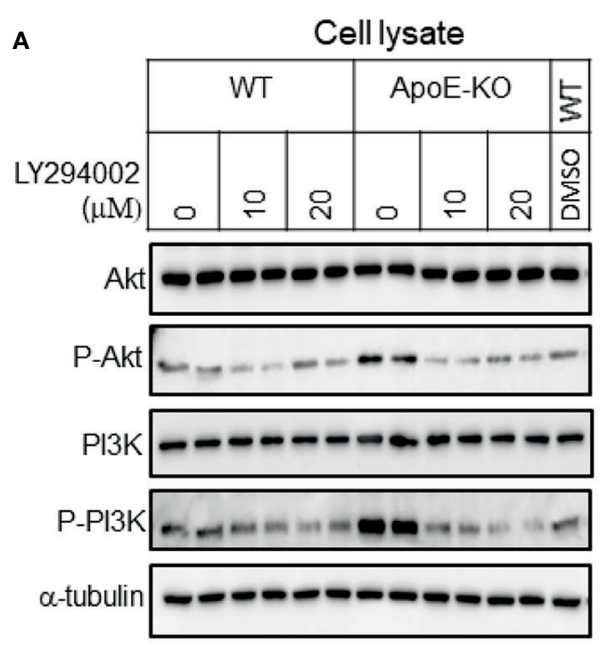

B

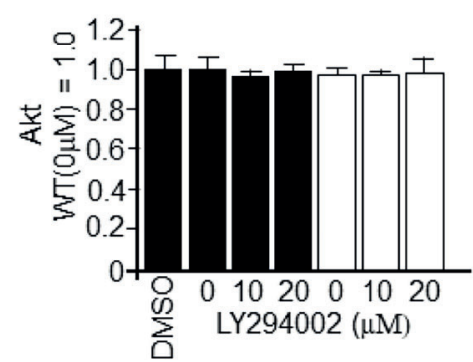

D

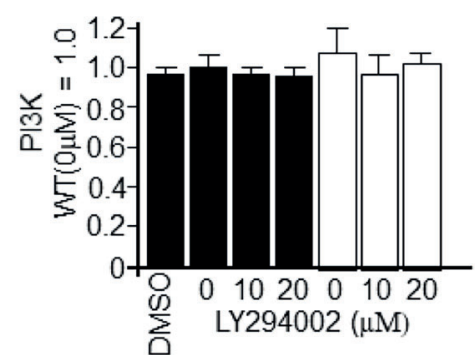

C

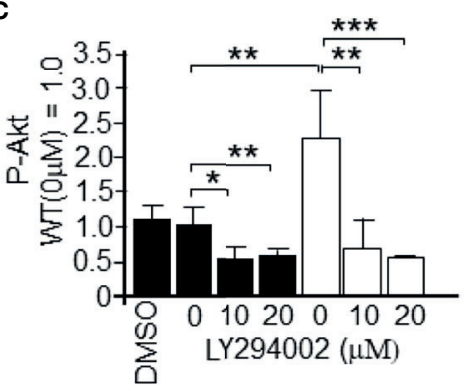

$\mathbf{E}$

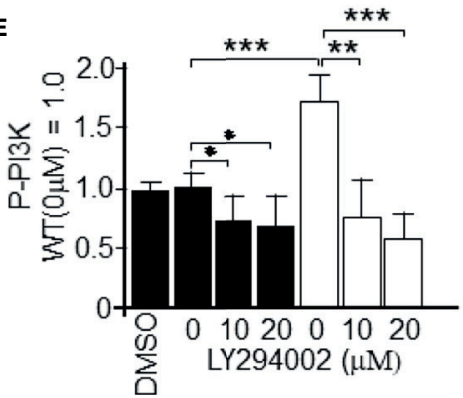

FIGURE 6 | Attenuation of the activation of PI3K and Akt in ApoE-deficient astrocytes by a PI3K-specific inhibitor, LY294002. Astrocyte cultures prepared from WT and ApoE-KO mouse brain were treated with LY294002, a specific inhibitor of PI3K. The cultures were then incubated for another 2 days, and the cell lysates were harvested. (A) An equal amount of protein from each sample was subjected to western blot analysis using antibodies against Akt, p-Akt, PI3K, p-PI3K, and $\alpha$-tubulin. (B-E) The intensity of each band was quantified by image analysis software (ImageJ 1.46r; Java 1.6.0-20 [64 bit]). Data are expressed as the mean \pm SE. $\mathrm{n}=3$ each. ${ }^{*} p<0.05,{ }^{\star *} p<0.01,{ }^{* *} p<0.001$ by one-way ANOVA followed by the Bonferroni-Dunn test.

was decreased in the CSF and serum isolated from the WT mice that were fed the high-fat diet and had significantly increased cholesterol levels in the serum and brain. These lines of evidence suggest that increased levels of cholesterol in ApoE-deficient astrocytes stimulate the PI3K/Akt pathway, which in turn attenuates exosome release.

With respect to the increased levels of brain cholesterol in ApoE-deficient astrocytes, it may be possible that the cause was a lack of ApoE-mediated cholesterol efflux, as our previous study has shown (22). In support of this notion, it is known that ApoE is an important modulator of atherosclerosis as it reduces cholesterol accumulation in vessels, and spontaneous hyperlipidemia and atherosclerosis have been observed in mice lacking ApoE (30, 31).

The next question to be addressed was whether the attenuated exosome release and the accompanying enhancement of PI3K and Akt phosphorylation were caused by increased levels of cellular cholesterol, but not by the absence of ApoE. To answer this question, we treated cells with cholesterol and analyzed the levels of released exosomes, as well as PI3K and Akt phosphorylation. We found that the addition of cholesterol to astrocytes increased the levels of PI3K and Akt phosphorylation, 
A

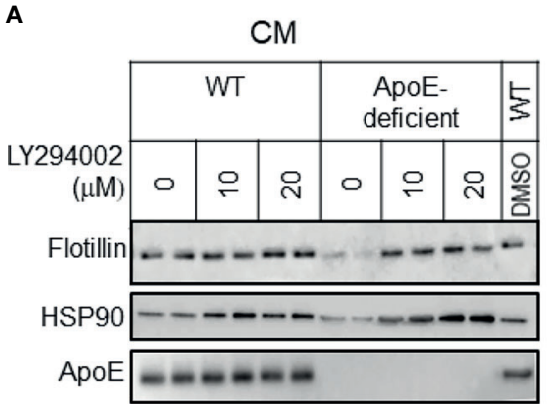

C

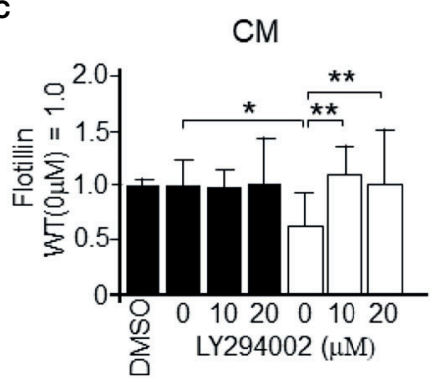

D

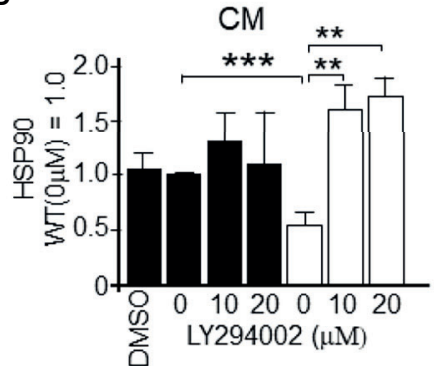

B

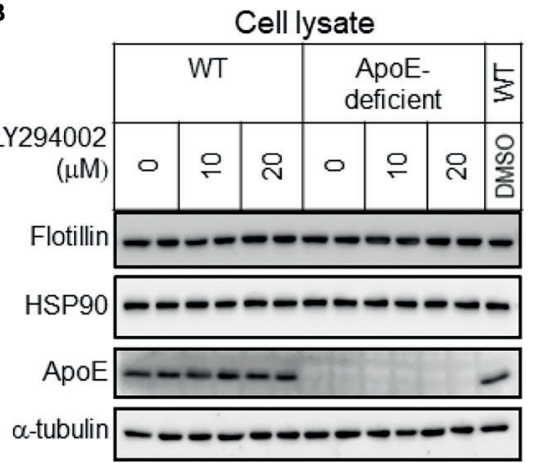

E
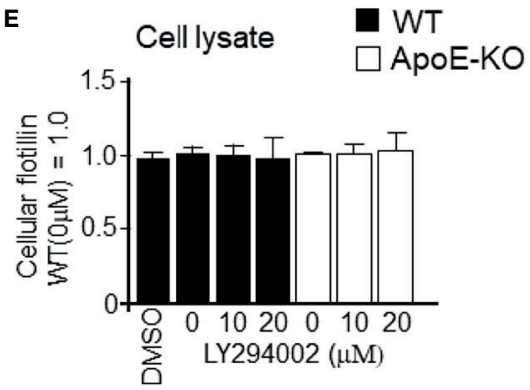

$\mathbf{F}$

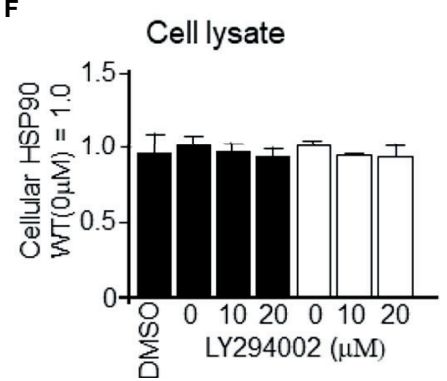

FIGURE 7 | Recovery of the decreased exosome release by treatment with a PI3K-specific inhibitor, LY294002, in cultured ApoE-deficient astrocytes. Astrocyte cultures prepared from WT and ApoE-KO mouse brain were treated with LY294002, a specific inhibitor of PI3K. The cultures were then incubated for another 2 days, and the CM and cell lysates were harvested. (A, B) Western blot analysis of each sample was performed to determine the levels of flotillin, HSP90, and ApoE in the CM and cell lysates from the WT and ApoE-deficient astrocytes. (C-F) The intensity of each band was quantified by image analysis software (Image J 1.46r; Java 1.6.0-20 [64 bit]). Data are expressed as the mean \pm SE. $n=3$ each. ${ }^{*} p<0.05,{ }^{* *} p<0.01,{ }^{* * *} p<0.001$ by one-way ANOVA followed by the Bonferroni-Dunn test.

and the cellular cholesterol levels significantly increased (Figures 2 and 3). In contrast, reducing the cellular cholesterol levels by $\beta$-CD treatment significantly enhanced exosome release and reduced the levels of phosphorylated PI3K/Akt (Figures 4, 5). These results indicated that the increased levels of cholesterol, but not another function of ApoE, regulate the exosome release accompanied by enhanced phosphorylation of PI3K and Akt. As noted above, exosomes are rich in cholesterol, SM, glycosphingolipids, and phosphatidylserine, which are essential players in exosome biogenesis and release (7). Lipids, such as cholesterol, accumulate in intracellular vesicles under certain pathological conditions, such as Alzheimer's disease and lysosomal storage disorders $(32,33)$. It has been reported that cholesterol regulates vesicular fusion with the plasma membrane, a step that is assumed to be critical for exosome release by controlling the content of specific proteins (34). However, no direct evidence showing the involvement of cholesterol in exosome release has yet been shown. Regarding lipid-mediated exosome release, there have been reports showing that exosome release is modulated in a sphingolipid-dependent manner (19), and that the activation of sphingosine 1-phosphate receptors mediates the maturation of exosomes (35). In addition to sphingolipid, in the present study, we showed for the first time the cellular cholesterol-dependent regulation of exosome release.

Next, we examined whether the cholesterol-dependent regulation of exosome release is mediated by $\mathrm{PI} 3 \mathrm{~K} / \mathrm{Akt}$ activity using a specific inhibitor of PI3K, LY294002, in cultured WT and ApoE-deficient astrocytes. We found that the reduced levels of exosome release in ApoE-deficient astrocytes recovered to levels similar to those in WT astrocytes (Figure 7C). These results 

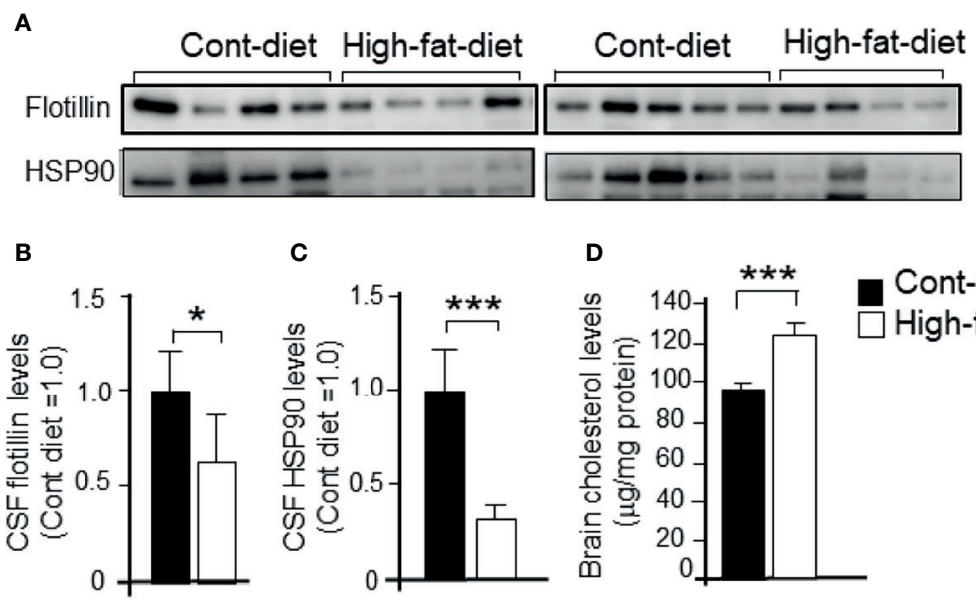

C
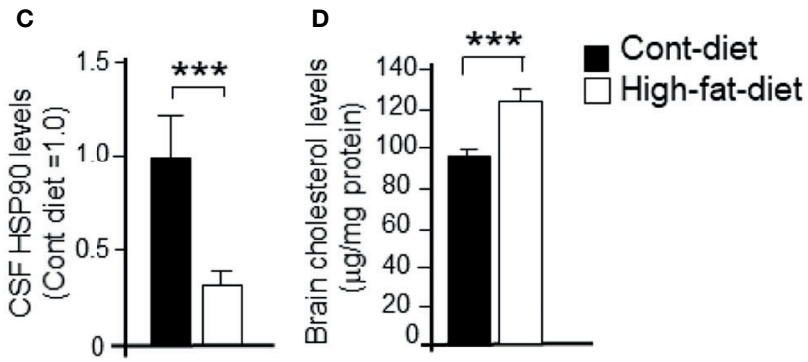

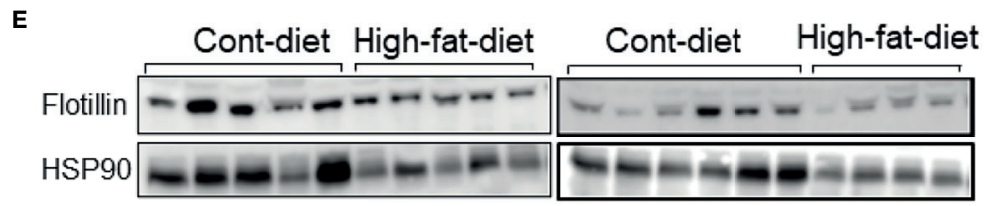

F

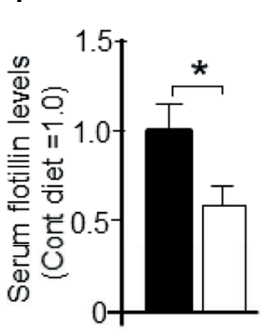

G

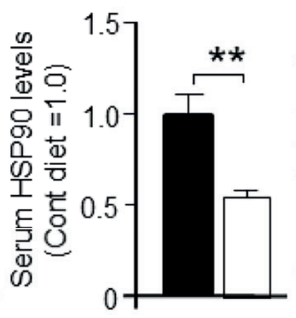

H

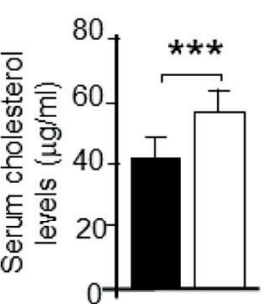

I

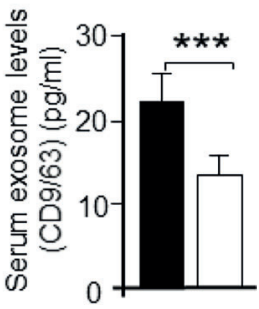

J

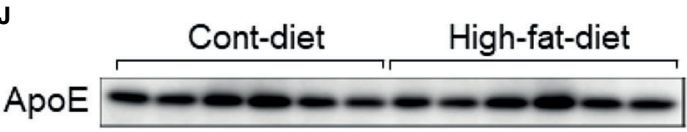

K

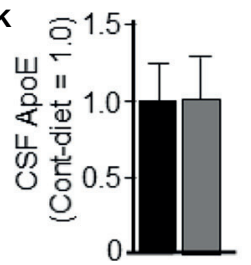

FIGURE 8 | Exosome levels in the CSF and serum collected from mice that were fed a high-fat diet or control diet. Three-month-old WT mice were fed a high-fat diet or control diet for 4 months. At the age of 7 months, the mice were sacrificed, CSF was obtained from the cisterna magna, and serum was collected. Then, each sample was subjected to western blot analysis. (A) CSF samples were subjected to western blot analysis using antibodies against flotillin and HSP90. (B, C) The intensity of each band representing CSF flotillin and HSP90 was quantified by image analysis software (ImageJ 1.46r; Java 1.6.0-20 [64 bit]). (D) The cholesterol levels in mouse brain were determined by a cholesterol determination kit. (E) Serum samples were subjected to western blot analysis using antibodies against flotillin and HSP90. (F, G) The intensity of each band representing serum flotillin and HSP90 was quantified by image analysis software (ImageJ 1.46r; Java 1.6.0-20 [64 bit]). (H) The serum cholesterol concentrations were determined by a cholesterol determination kit. (I) Serum exosome levels were determined by a CD9/CD63 ELISA kit as described in the Experimental Procedures section. $\mathbf{( J , ~ K ) ~ A p o E ~ l e v e l s ~ i n ~ t h e ~ C S F ~ c o l l e c t e d ~ f r o m ~ m i c e ~ f e d ~ w i t h ~ c o n t r o l ~ d i e t ~ a n d ~ h i g h - f a t ~ d i e t ~ w e r e ~ d e t e r m i n e d ~ b y ~}$ western blot analysis using anti-ApoE antibody. Data are expressed as the mean \pm SE. ${ }^{\star} p<0.05,{ }^{\star \star} p<0.01,{ }^{\star \star \star} p<0.001$ by Student's $t$-test.

indicated that the PI3K/Akt pathway mediates the cholesteroldependent reduction in the levels of exosome release from cultured astrocytes. Although the cholesterol-dependent regulation of PI3K/Akt activity has not been previously reported in neural cells, in agreement with our present results, previous studies in non-neuronal cells have shown that cholesterol activates PI3K/Akt signaling in human bone marrow mesenchymal stromal cells (36) and in human melanoma cells $(37,38)$. In contrast, cholesterol depletion in lipid rafts of cell membranes due to methylated $\beta$-CD treatment induces apoptosis through the PI3K-Akt pathway in nonneuronal cell lines (39). Although the mechanisms underlying exosome formation and release are poorly understood, it is believed that several pathways and molecules are involved in exosome biogenesis and release (40). Our findings suggest that involvement of the PI3K/Akt pathway is one of them. 
TABLE 1 | Basic formulation of high-fat diet and control diet.

\begin{tabular}{|c|c|c|}
\hline Basic formulation & $\begin{array}{c}\text { High-fat diet (HFD-60) } \\
\text { (g/kg diet) }\end{array}$ & $\begin{array}{c}\text { Control diet (AIN-93M) } \\
\text { (g/kg diet) }\end{array}$ \\
\hline Milk Casein & 256.0 & 140.0 \\
\hline L-Cystine & 3.6 & 1.8 \\
\hline Maltodextrin & 60.0 & 0.0 \\
\hline Cornstarch & 0.0 & 465.692 \\
\hline$\alpha$-Cornstarch & 160.0 & 155.0 \\
\hline Sucrose & 55.0 & 100.0 \\
\hline Soybean oil & 20.0 & 4.0 \\
\hline Lard & 330.0 & 0.0 \\
\hline Powdered Cellulose & 66.1 & 50.0 \\
\hline mineral mix & 35.0 & 35.0 \\
\hline Calcium carbonate & 1.8 & 0.0 \\
\hline Vitamin mix & 10.0 & 10.0 \\
\hline Choline bitartrate & 2.5 & 2.5 \\
\hline tert-Butylhydroquinone & 0.0 & 0.008 \\
\hline Total & $1,000.0 \mathrm{~g}$ & $1,000.0 \mathrm{~g}$ \\
\hline
\end{tabular}

Taken together, we showed that the level of cellular cholesterol regulates exosome release by modulating the PI3K/ Akt pathway. The role and function of exosomes in brain are not well understood; however, several reports have showed that flotillin plays a role in axonal regeneration $(41,42)$. There are also studies reporting the involvement of flotillin in neurodegenerative diseases such as Alzheimer's disease (43) and Parkinson's disease (44). Although the roles of exosomes in diseases and the maintenance of cellular functions are not well understood, our findings from the present study provide new insights into the molecular mechanisms that regulate exosome release from cells.

\section{CONCLUSION}

In summary, we found that cellular cholesterol regulates exosome release from astrocytes in culture; that is higher levels of cholesterol attenuates exosome release and lower levels of cholesterol enhances exosome release. These cholesteroldependent regulation of exosome release is mediated by PI3K/ Akt pathways. The cholesterol-dependent regulation of exosome release in the CSF and serum was also confirmed by in vivo experiments. These results suggest that exosome release is

\section{REFERENCES}

1. Yanez-Mo M, Siljander PR, Andreu Z, Zavec AB, Borras FE, Buzas EI, et al. Biological Properties of Extracellular Vesicles and Their Physiological Functions. J Extracell Vesicles (2015) 4:27066. doi: 10.3402/jev.v4.27066

2. van Niel G, D'Angelo G, Raposo G. Shedding Light on the Cell Biology of Extracellular Vesicles. Nat Rev Mol Cell Biol (2018) 19(4):213-28. doi: 10.1038/ nrm.2017.125

3. Thery C, Ostrowski M, Segura E. Membrane Vesicles as Conveyors of Immune Responses. Nat Rev Immunol (2009) 9(8):581-93. doi: 10.1038/ nri2567

4. Pisitkun T, Shen RF, Knepper MA. Identification and Proteomic Profiling of Exosomes in Human Urine. Proc Natl Acad Sci U.S.A. (2004) 101(36):1336873. doi: 10.1073/pnas.0403453101

5. Laulagnier K, Motta C, Hamdi S, Roy S, Fauvelle F, Pageaux JF, et al. Mast Cell- and Dendritic Cell-Derived Exosomes Display a Specific Lipid regulated by cellular cholesterol via stimulation of the PI3K/ Akt signal pathway.

\section{DATA AVAILABILITY STATEMENT}

The original contributions presented in the study are included in the article/supplementary material. Further inquiries can be directed to the corresponding author.

\section{ETHICS STATEMENT}

The animal study was reviewed and approved by Nagoya City University Animal Ethics Committee.

\section{AUTHOR CONTRIBUTIONS}

MM designed research. MA, TN, TF, YG, YC and KZ performed research. MA, TN, KZ and MM analyzed data. MA, TN and MM wrote the paper. All authors contributed to the article and approved the submitted version.

\section{FUNDING}

This work was supported by Grant-in-Aid for Scientific Research B (16H05559) (to MM) and for Challenging Exploratory Research (15K15712) (to MM) from the Ministry of Education, Culture, Sports, Science and Technology, Japan. This study was also supported by AMED "Project of Translational and Clinical Research Seed A" under grant number A-128, and "Research and Development Grants for Dementia” under grant number 21dk0207050h0002 (to MM).

\section{ACKNOWLEDGMENTS}

We thank all study participants for their cooperation.

Composition and an Unusual Membrane Organization. Biochem J (2004) 380(Pt 1):161-71. doi: 10.1042/BJ20031594

6. de Gassart A, Geminard C, Fevrier B, Raposo G, Vidal M. Lipid RaftAssociated Protein Sorting in Exosomes. Blood (2003) 102(13):4336-44. doi: 10.1182/blood-2003-03-0871

7. Skotland T, Hessvik NP, Sandvig K, Llorente A. Exosomal Lipid Composition and the Role of Ether Lipids and Phosphoinositides in Exosome Biology. J Lipid Res (2019) 60(1):9-18. doi: 10.1194/jlr.R084343

8. Ratajczak J, Miekus K, Kucia M, Zhang J, Reca R, Dvorak P, et al. Embryonic Stem Cell-Derived Microvesicles Reprogram Hematopoietic Progenitors: Evidence for Horizontal Transfer of mRNA and Protein Delivery. Leukemia (2006) 20(5):847-56. doi: 10.1038/sj.leu.2404132

9. Valadi H, Ekstrom K, Bossios A, Sjostrand M, Lee JJ, Lotvall JO. ExosomeMediated Transfer of mRNAs and microRNAs is a Novel Mechanism of Genetic Exchange Between Cells. Nat Cell Biol (2007) 9(6):654-9. doi: $10.1038 /$ ncb1596 
10. Skog J, Wurdinger T, van Rijn S, Meijer DH, Gainche L, Sena-Esteves M, et al. Glioblastoma Microvesicles Transport RNA and Proteins That Promote Tumour Growth and Provide Diagnostic Biomarkers. Nat Cell Biol (2008) 10(12):1470-6. doi: 10.1038/ncb1800

11. Raposo G, Nijman HW, Stoorvogel W, Liejendekker R, Harding CV, Melief CJ, et al. B Lymphocytes Secrete Antigen-Presenting Vesicles. J Exp Med (1996) 183(3):1161-72. doi: 10.1084/jem.183.3.1161

12. Abdullah M, Kimura N, Akatsu H, Hashizume Y, Ferdous T, Tachita T, et al. Flotillin is a Novel Diagnostic Blood Marker of Alzheimer's Disease. J Alzheimers Dis (2019) 72(4):1165-76. doi: 10.3233/JAD-190908

13. Admyre C, Johansson SM, Qazi KR, Filen JJ, Lahesmaa R, Norman M, et al. Exosomes With Immune Modulatory Features are Present in Human Breast Milk. J Immunol (2007) 179(3):1969-78. doi: 10.4049/jimmunol.179.3.1969

14. Abdullah M, Takase H, Nunome M, Enomoto H, Ito J, Gong JS, et al. Amyloid-Beta Reduces Exosome Release From Astrocytes by Enhancing JNK Phosphorylation. J Alzheimers Dis (2016) 53(4):1433-41. doi: 10.3233/JAD160292

15. Yuana Y, Sturk A, Nieuwland R. Extracellular Vesicles in Physiological and Pathological Conditions. Blood Rev (2013) 27(1):31-9. doi: 10.1016/j.blre. 2012.12.002

16. Tamai K, Tanaka N, Nakano T, Kakazu E, Kondo Y, Inoue J, et al. Exosome Secretion of Dendritic Cells is Regulated by Hrs, an ESCRT-0 Protein. Biochem Biophys Res Commun (2010) 399(3):384-90. doi: 10.1016/ j.bbrc.2010.07.083

17. Colombo M, Moita C, van Niel G, Kowal J, Vigneron J, Benaroch P, et al. Analysis of ESCRT Functions in Exosome Biogenesis, Composition and Secretion Highlights the Heterogeneity of Extracellular Vesicles. J Cell Sci (2013) 126(Pt 24):5553-65. doi: 10.1242/jcs.128868

18. Subra C, Grand D, Laulagnier K, Stella A, Lambeau G, Paillasse M, et al. Exosomes Account for Vesicle-Mediated Transcellular Transport of Activatable Phospholipases and Prostaglandins. J Lipid Res (2010) 51 (8):2105-20. doi: 10.1194/jlr.M003657

19. Yuyama K, Sun H, Mitsutake S, Igarashi Y. Sphingolipid-Modulated Exosome Secretion Promotes Clearance of Amyloid-Beta by Microglia. J Biol Chem (2012) 287(14):10977-89. doi: 10.1074/jbc.M111.324616

20. Yuyama K, Sun H, Mikami D, Mioka T, Mukai K, Igarashi Y. LysosomalAssociated Transmembrane Protein 4B Regulates Ceramide-Induced Exosome Release. FASEB J (2020) 34(12):16022-33. doi: 10.1096/ fj.202001599R

21. Hatters DM, Peters-Libeu CA, Weisgraber KH. Apolipoprotein E Structure: Insights Into Function. Trends Biochem Sci (2006) 31(8):445-54. doi: 10.1016/ j.tibs.2006.06.008

22. Michikawa M, Fan QW, Isobe I, Yanagisawa K. Apolipoprotein E Exhibits Isoform-Specific Promotion of Lipid Efflux From Astrocytes and Neurons in Culture. J Neurochem (2000) 74(3):1008-16. doi: 10.1046/j.1471-4159. 2000.0741008.x

23. Pitas RE, Boyles JK, Lee SH, Hui D, Weisgraber KH. Lipoproteins and Their Receptors in the Central Nervous System. Characterization of the Lipoproteins in Cerebrospinal Fluid and Identification of Apolipoprotein B, E(LDL) Receptors in the Brain. J Biol Chem (1987) 262(29):14352-60. doi: 10.1016/S0021-9258(18)47945-8

24. Krimbou L, Denis M, Haidar B, Carrier M, Marcil M, Genest JJr. Molecular Interactions Between apoE and ABCA1: Impact on apoE Lipidation. J Lipid Res (2004) 45(5):839-48. doi: 10.1194/jlr.M300418-JLR200

25. Ito J, Zhang LY, Asai M, Yokoyama S. Differential Generation of HighDensity Lipoprotein by Endogenous and Exogenous Apolipoproteins in Cultured Fetal Rat Astrocytes. J Neurochem (1999) 72(6):2362-9. doi: 10.1046/j.1471-4159.1999.0722362.x

26. Gong JS, Kobayashi M, Hayashi H, Zou K, Sawamura N, Fujita SC, et al. Apolipoprotein E (ApoE) Isoform-Dependent Lipid Release From Astrocytes Prepared From Human ApoE3 and ApoE4 Knock-in Mice. J Biol Chem (2002) 277(33):29919-26. doi: 10.1074/jbc.M203934200

27. Minagawa H, Watanabe A, Akatsu H, Adachi K, Ohtsuka C, Terayama Y, et al. Homocysteine, Another Risk Factor for Alzheimer Disease, Impairs Apolipoprotein E3 Function. J Biol Chem (2010) 285(49):38382-8. doi: 10.1074/ jbc.M110.146258

28. Ju R, Zhuang ZW, Zhang J, Lanahan AA, Kyriakides T, Sessa WC, et al. Angiopoietin-2 Secretion by Endothelial Cell Exosomes: Regulation by the
Phosphatidylinositol 3-Kinase (PI3K)/Akt/endothelial Nitric Oxide Synthase (eNOS) and Syndecan-4/Syntenin Pathways. J Biol Chem (2014) 289(1):5109. doi: 10.1074/jbc.M113.506899

29. Mollinedo F, Gajate C. Lipid Rafts as Signaling Hubs in Cancer Cell Survival/ Death and Invasion: Implications in Tumor Progression and Therapy: Thematic Review Series: Biology of Lipid Rafts. J Lipid Res (2020) 61 (5):611-35. doi: 10.1194/jlr.TR119000439

30. Zhang SH, Reddick RL, Piedrahita JA, Maeda N. Spontaneous Hypercholesterolemia and Arterial Lesions in Mice Lacking Apolipoprotein E. Science (1992) 258(5081):468-71. doi: 10.1126/science.1411543

31. Gaudreault N, Kumar N, Posada JM, Stephens KB, Reyes de Mochel NS, Eberle D, et al. ApoE Suppresses Atherosclerosis by Reducing Lipid Accumulation in Circulating Monocytes and the Expression of Inflammatory Molecules on Monocytes and Vascular Endothelium. Arterioscler Thromb Vasc Biol (2012) 32 (2):264-72. doi: 10.1161/ATVBAHA.111.238964

32. Distl R, Meske V, Ohm TG. Tangle-Bearing Neurons Contain More Free Cholesterol Than Adjacent Tangle-Free Neurons. Acta Neuropathol (2001) 101(6):547-54. doi: 10.1007/s004010000314

33. Kuech EM, Brogden G, Naim HY. Alterations in Membrane Trafficking and Pathophysiological Implications in Lysosomal Storage Disorders. Biochimie (2016) 130:152-62. doi: 10.1016/j.biochi.2016.09.011

34. Ammar MR, Kassas N, Chasserot-Golaz S, Bader MF, Vitale N. Lipids in Regulated Exocytosis: What are They Doing? Front Endocrinol (Lausanne) (2013) 4:125. doi: 10.3389/fendo.2013.00125

35. Kajimoto T, Okada T, Miya S, Zhang L, Nakamura S. Ongoing Activation of Sphingosine 1-Phosphate Receptors Mediates Maturation of Exosomal Multivesicular Endosomes. Nat Commun (2013) 4:2712. doi: 10.1038/ncomms3712

36. Baker N, Sohn J, Tuan RS. Promotion of Human Mesenchymal Stem Cell Osteogenesis by PI3-Kinase/Akt Signaling, and the Influence of Caveolin-1/ Cholesterol Homeostasis. Stem Cell Res Ther (2015) 6:238. doi: 10.1186/ s13287-015-0225-8

37. Yamauchi Y, Furukawa K, Hamamura K. Positive Feedback Loop Between PI3K-Akt-Mtorc1 Signaling and the Lipogenic Pathway Boosts Akt Signaling: Induction of the Lipogenic Pathway by a Melanoma Antigen. Cancer Res (2011) 71(14):4989-97. doi: 10.1158/0008-5472.CAN-10-4108

38. Xu J, Qian J, Xie X, Lin L, Ma J, Huang Z, et al. High Density Lipoprotein Cholesterol Promotes the Proliferation of Bone-Derived Mesenchymal Stem Cells via Binding Scavenger Receptor-B Type I and Activation of PI3K/Akt, MAPK/ERK1/2 Pathways. Mol Cell Biochem (2012) 371(1-2):55-64. doi: $10.1007 / \mathrm{s} 11010-012-1422-8$

39. Motoyama K, Kameyama K, Onodera R, Araki N, Hirayama F, Uekama K, et al. Involvement of PI3K-Akt-Bad Pathway in Apoptosis Induced by 2,6-DiO-Methyl-Beta-Cyclodextrin, Not 2,6-Di-O-Methyl-Alpha-Cyclodextrin, Through Cholesterol Depletion From Lipid Rafts on Plasma Membranes in Cells. Eur J Pharm Sci (2009) 38(3):249-61. doi: 10.1016/j.ejps.2009.07.010

40. Hessvik NP, Llorente A. Current Knowledge on Exosome Biogenesis and Release. Cell Mol Life Sci (2018) 75(2):193-208. doi: 10.1007/s00018-017-2595-9

41. Schulte T, Paschke KA, Laessing U, Lottspeich F, Stuermer CA. Reggie-1 and Reggie-2, Two Cell Surface Proteins Expressed by Retinal Ganglion Cells during Axon Regeneration. Development (1997) 124(2):577-87. doi: 10.1242/ dev.124.2.577

42. Stuermer CA. How Reggies Regulate Regeneration and Axon Growth. Cell Tissue Res (2012) 349(1):71-7. doi: 10.1007/s00441-012-1343-6

43. Girardot N, Allinquant B, Langui D, Laquerriere A, Dubois B, Hauw JJ, et al. Accumulation of Flotillin-1 in Tangle-Bearing Neurones of Alzheimer's Disease. Neuropathol Appl Neurobiol (2003) 29(5):451-61. doi: 10.1046/ j.1365-2990.2003.00479.x

44. Jacobowitz DM, Kallarakal AT. Flotillin-1 in the Substantia Nigra of the Parkinson Brain and a Predominant Localization in Catecholaminergic Nerves in the Rat Brain. Neurotox Res (2004) 6(4):245-57. doi: 10.1007/BF03033435

Conflict of Interest: The authors declare that the research was conducted in the absence of any commercial or financial relationships that could be construed as a potential conflict of interest.

Publisher's Note: All claims expressed in this article are solely those of the authors and do not necessarily represent those of their affiliated organizations, or those of the publisher, the editors and the reviewers. Any product that may be evaluated in 
this article, or claim that may be made by its manufacturer, is not guaranteed or endorsed by the publisher.

Copyright $\odot 2021$ Abdullah, Nakamura, Ferdous, Gao, Chen, Zou and Michikawa. This is an open-access article distributed under the terms of the Creative Commons
Attribution License (CC BY). The use, distribution or reproduction in other forums is permitted, provided the original author $(s)$ and the copyright owner(s) are credited and that the original publication in this journal is cited, in accordance with accepted academic practice. No use, distribution or reproduction is permitted which does not comply with these terms. 